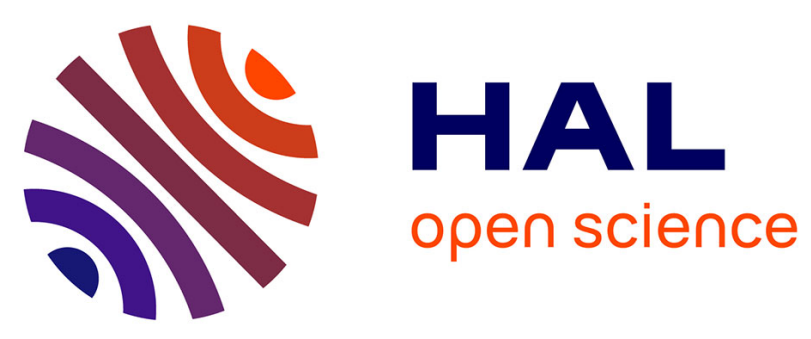

\title{
Sur le mouvement lumineux
}

\author{
M. Gouy
}

\section{To cite this version:}

M. Gouy. Sur le mouvement lumineux. J. Phys. Theor. Appl., 1886, 5 (1), pp.354-362. 10.1051/jphystap:018860050035401 . jpa-00238657

\section{HAL Id: jpa-00238657 https://hal.science/jpa-00238657}

Submitted on 1 Jan 1886

HAL is a multi-disciplinary open access archive for the deposit and dissemination of scientific research documents, whether they are published or not. The documents may come from teaching and research institutions in France or abroad, or from public or private research centers.
L'archive ouverte pluridisciplinaire HAL, est destinée au dépôt et à la diffusion de documents scientifiques de niveau recherche, publiés ou non, émanant des établissements d'enseignement et de recherche français ou étrangers, des laboratoires publics ou privés. 


\section{SUR LE MOUVEMENT LUMINEUX;}

Par M. GOUY.

1. Préliminaires. - Ce Mémoire a pour objet l'étude théorique des effets produits par les mouvements complexes qui constituent les rayons lumineux réels. On sait que la théorie ondulatoire, dans les explications qu'elle donne des phénomènes optiques, a pour objet immédiat le mouvement simple, dans lequel la vitesse vibratoire $v$ en un point quelconque est donnée par une équation de la forme

$$
v=a \sin 2 \pi\left(\frac{t}{\theta}+b\right)
$$

$t$ désignant le temps, $a, b$ et $\theta$ des constantes. Cette équation définit une suite entièrement illimitée de vibrations pendulaires, d'une régularité absolue, dont la période est $\theta$.

Si ces conditions de régularité et de durée ne se trouvent pas rigoureusement réalisées, l'équation du mouvement est différente, et, par suite, un problème nouveau se trouve posé, la solution fournie par la théorie pour un mouvement simple n'étant plus applicable en général. S'il s'agit, par exemple, d'un phénomène d'interférences ou de diffraction, on voit immédiatement que toute irrégularité ou toute interruption entraine une perturbation dans l'effet produit, comme on l'a remarqué depuis longtemps. Il en sera encore de même, abstraction faite des interférences, toutes les fois que l'on aura à considérer des milieux doués de dispersion. En effet, le mouvement vibratoire dans les divers milieux devant toujours satisfaire aux équations différentielles des petits

( $\left.{ }^{1}\right)$ Vogel, Sitzungsberichte, 10 July 1879 , 22 Feb. I88o. 
mouvements de ces milieux, il n'est nullement permis de faire subir une altération, quelle qu'elle soit, au mouvement vibratoire (I), et de supposer ensuite que ce mouvement se comportera, dans les milieux doués de dispersion, comme s'il n'était pas altéré. Ainsi, par exemple, on n'est pas en droit de supposer que le mouvement ( $\mathrm{I}$ ) ne comprend qu'un nombre de vibrations limité, et qu'il se propagera dans les divers milieux comme s'il formait la suite entièrement illimitée que définit l'équation (I).

D'autre part, on a remarqué depuis longtemps qu'aucune source lumineuse ne peut produire une série de vibrations indéfinie et parfaitement régulière, ne fût-ce qu'en raison du renouvellement incessant des particules incandescentes. Ainsi aucun mouvement lumineux réel, même le moins complexe qu'on puisse supposer, ne rentre rigoureusement dans le cas du mouvement simple que considère la théorie ondulatoire.

Cette difficulté s'est présentée dès l'origine de cette théorie. On y répond d'ordinaire en supposant que les sources lumineuses produisent des séries de vibrations régulières, mais troublées de temps à autre par des perturbations subites ou de courte durée. Si la série, entre deux perturbations, comprend un grand nombre de vibrations, on peut prévoir que l'effet moyen d'un pareil mouvement différera peu de celui d'un mouvement simple. Mais cet aperçu, reposant sur une hypothèse, ne peut servir de base à une étude rationnelle du problème qui nous occupe, et nous verrons que, pour les sources donnant des spectres continus, on peut se faire une idée beaucoup moins étroite du mouvement lumineux. De plus, cet aperçu ne nous apprend rien sur les effets des perturbations elles-mêmes, qui paraissent jouer un rôle important dans la constitution des spectres fournis par les vapeurs et les gaz incandescents.

Pour traiter la question à un point de vue général, nous laisserons entièrement arbitraire le mouvement lumineux incident. A l'aide de la formule de Fourier, nous représenterons ce mouvement par la superposition de mouvements simples, tels que (I), pour lesquels nous savons calculer l'effet de l'appareil ou du système optique que nous voulons étudier. D'après le principe de la superposition des petits mouvements, chacun de ces mouvements simples se comportera comme s'il existait seul, et leur superposi- 
tion formera le mouvement réel, dont il ne restera qu'à discuter l'expression. Pour ne pas compliquer cette discussion, nous laisserons de côté pour le moment les questions qui se rattachent à la constitution de la lumière naturelle, au point de vue de la polarisation, et nous admettrons que les rayons que nous étudions sont polarisés rectilignement.

2. Formules générales. - Pour fixer les idées, nous considérerons des rayons incidents parallèles.

Soit un plan fixe M normal à ces rayons, à l'entrée du système optique donné. Soit

$$
v=f(t)
$$

la vitesse vibratoire sur le plan $M$. La fonction $f(t)$ définit le mouvement incident, et nous allons en déduire les effets du système optique donné.

Nous considérerons le mouvement lumineux pendant un intervalle de temps $2 \mathrm{~T}$, pris arbitrairement. Prenons pour origine du temps le commencement de cet intervalle. Pour toutes les valeurs de $t$ comprises entre zéro et $2 \mathrm{~T}$, nous avons, par la formule de Fourier,

$$
f(t)=\mathrm{Q}+\frac{\mathrm{I}}{\mathrm{T}} \sum_{1}^{\infty}\left(\mathrm{A}_{n} \sin \frac{n \pi t}{\mathrm{~T}}+\mathrm{B}_{n} \cos \frac{n \pi t}{\mathrm{~T}}\right),
$$

en posant

$$
\left\{\begin{array}{l}
\mathrm{Q}=\frac{\mathrm{T}}{2 \mathrm{~T}} \int_{0}^{2 \mathrm{~T}} f(t) d t \\
\mathrm{\Lambda}_{n}=\int_{0}^{2 \mathrm{~T}} f(t) \sin \frac{n \pi t}{\mathrm{~T}} d t \\
\mathrm{~B}_{n}=\int_{0}^{2 \mathrm{~T}} f(t) \cos \frac{n \pi t}{\mathrm{~T}} d t .
\end{array}\right.
$$

Dans la formule (3), la sommation s'étend à toutes les valeurs entières et positives de $n$. Le terme $\mathrm{Q}$ exprime la distance des deux positions qu'occupe une particule d'éther sur le plan M aux temps zéro et $2 \mathrm{~T}$, divisée par $2 \mathrm{~T}$; ce terme sera toujours négligeable lorsque $2 \mathrm{~T}$ sera très grand, et nous n'en tiendrons pas compte.

Il est essentiel de remarquer que le second membre de (3), que 
nous désignerons par $\mathrm{F}(t)$, n'est identique à $f(t)$ que dans l'intervalle de zéro à $2 \mathrm{~T}$, et reprend périodiquement les mêmes valeurs en dehors de cet intervalle. Nous prendrons $\mathrm{F}(t)$ comme point de départ des calculs ultérieurs, et nous substituerons ainsi au véritable mouvement incident $f(t)$ un autre mouvement qui lui est identique dans l'intervalle considéré, de zéro à $2 \mathrm{~T}$, et qui peut en différer en dehors de cet intervalle.

Si $2 \mathrm{~T}$ est très grand, l'effet produit pendant cet intervalle par le mouvement incident ne peut dépendre que de la nature actuelle de ce mouvement, et non de ce qu'il était avant l'intervalle considéré, ou de ce qu'il sera après cet intervalle. Nous pourrons donc calculer cet effet en considérant $F(t)$ comme le mouvement incident. Cela n'est pas évident toutefois au début et à la fin de l'intervalle considéré, où le résultat pourra être inexact pendant un temps très court. Si le système optique donné ne comprend pas de milieux doués de dispersion, ce temps est précisément égal à celui que met la lumière à aller du plan $\mathbf{M}$ au point considéré. Dans tous les cas, cette durée sera insensible vis-à-vis de ${ }_{2} \mathbf{T}$ dès que cet intervalle sera grand, et nous n'en tiendrons pas compte.

En posant

$$
\theta_{n}=\frac{2 \mathrm{~T}}{n}
$$

le second membre de (3) s'écrit

$$
\mathrm{F}(t)=\frac{1}{\mathrm{~T}} \sum_{1}^{\infty}\left(\Lambda_{n} \sin 2 \pi \frac{t}{\theta_{n}}+\mathrm{B}_{n} \cos 2 \pi \frac{t}{\theta_{n}}\right) .
$$

Ainsi le mouvement $\mathrm{F}(t)$ peut être regardé comme formé par la superposition de mouvements simples, dont l'équation générale est

$$
v_{n}=\frac{\mathbf{I}}{\mathrm{T}}\left(\mathrm{A}_{n} \sin 2 \pi \frac{t}{\theta_{n}}+\mathrm{B}_{n} \cos 2 \pi \frac{t}{\theta_{n}}\right)
$$

Ces mouvements sont d'une durée indéfinie et d'une régularité absolue, et nous pouvons leur appliquer immédiatement les formules de la théorie ondulatoire.

Considérons un point $\mathrm{P}$ quelconque, dans le système optique donné. Soit $v_{n}^{\prime}$ la vitesse vibratoire qui existerait au point $\mathrm{P}$ si le 
mouvement incident était le mouvement simple (6). Nous aurions

$$
v_{n}^{\prime}=?\left(\theta_{n}\right) \frac{\mathrm{I}}{\mathrm{T}}\left[\mathrm{A}_{n} \sin 2 \pi\left(\frac{t}{\theta_{n}}+\gamma_{n}\right)+\mathrm{B}_{n} \cos 2 \pi\left(\frac{t}{\theta_{n}}+\gamma_{n}\right)\right],
$$

les quantités $\varphi\left(\theta_{n}\right)$ et $\%_{n}$ étant des constantes calculées, en fonction de la période $\theta_{n}$ du mouvement simple, par les formules ordinaires de la théorie ondulatoire, appliquées au système optique donné. Ainsi $\varphi\left(\theta_{n}\right)$ désigne le rapport des amplitudes qui existeraient au point $\mathrm{P}$ el sur le plan $\mathrm{M}$, si le mouvement incident était un mouvement simple de période $\theta_{n}$.

La vitesse réelle $v^{\prime}$ au point $\mathbf{P}$ résultera de la superposition de toutes les vitesses telles que $v_{n}^{\prime}$,

$$
v^{\prime}=\frac{\mathrm{I}}{\mathrm{T}} \sum_{1}\left\{\vartheta\left(\theta_{n}\right)\left[\mathrm{A}_{n} \sin 2 \pi\left(\frac{t}{\theta_{n}}+\gamma_{n}\right)+\mathrm{B}_{n} \cos 2 \pi\left(\frac{t}{\theta_{n}}+\gamma_{n}\right)\right]\right\}
$$

et la force vive au point $\mathrm{P}$ et à l'instant $t$ sera exprimée par $v^{\prime 2}$.

3. Lumière constante. - La réduction de la formule (8) présente en général des difficultés insurmontables; mais le problème devient très simple si l'on considère les mouvements lumineux constants, c'est-à-dire ceux qui donnent des phénomènes indépendants du temps, ce qui est le cas ordinaire.

Si nous faisons passer les rayons émis par une source lumineuse constante à travers un système optique fixe quelconque, l'expérience montre que l'intensité lumineuse en un point quelconque est sensiblement constante, c'est-à-dire que si elle subit des variations, celles-ci sont assez rapides pour être insensibles à l'observateur, qui ne peut apprécier que l'intensité moyenne dans un intervalle comprenant nécessairement un grand nombre de vibrations.

Supposons que le mouvement considéré $\mathrm{F}(t)$ soit de cette nature; il nous suffira de connaître la force vive moyenne I ou intensité lumineuse au point $\mathrm{P}$. Nous avons

$$
\begin{aligned}
& \mathbf{I}=\frac{\mathrm{I}}{2 \mathrm{~T}} \int_{0}^{2 \mathrm{~T}} v^{\prime 2} d t \\
&=\frac{\mathrm{I}}{2 \mathrm{~T}^{3}} \int_{0}^{2 \mathrm{~T}}\left(\sum _ { 1 } ^ { \infty } \left\{\varphi ( \theta _ { n } ) \left[\mathrm{~A}_{n} \sin 2 \pi\left(\frac{t}{\theta_{n}}+\chi_{n}\right)\right.\right.\right. \\
& \\
&\left.\left.\left.\quad+\mathrm{B}_{n} \cos 2 \pi\left(\frac{t}{\theta_{n}^{-}}+\chi_{n}\right)\right]\right\}\right)^{2} d t .
\end{aligned}
$$


Cette intégrale définie est une somme de termes de la forme

$$
\begin{aligned}
\int_{0}^{2 \mathrm{~T}}\left\{\varphi\left(\theta_{n}\right)\left[\mathrm{A}_{n} \sin 2 \pi\left(\frac{t}{\theta_{n}}+\chi_{n}\right)+\mathrm{B}_{n} \cos 2 \pi\left(\frac{t}{\sigma_{n}}+\gamma_{n}\right)\right]\right. \\
\left.\quad \times \varphi\left(\theta_{n^{\prime}}\right)\left[\Lambda_{n^{\prime}} \sin 2 \pi\left(\frac{t}{\theta_{n^{\prime}}}+\chi_{n^{\prime}}\right)+\mathrm{B}_{n^{\prime}} \cos 2 \pi\left(\frac{t}{\theta_{n^{\prime}}}+\chi_{n^{\prime}}\right)\right]\right\} d t .
\end{aligned}
$$

En remplaçant $\theta_{n}$ et $\theta_{n^{\prime}}$ par leurs valeurs $\frac{2 \mathrm{~T}}{n}$ et $\frac{2 \mathrm{~T}}{n^{\prime}}$, on voit aisć; ment que l'intégrale est égale à zéro pour $n \leqslant n^{\prime}$, et à

$$
\mathbf{T}\left(\mathbf{A}_{n}^{2}+\mathbf{B}_{n}^{2}\right)\left[\varphi\left(\theta_{n}\right)\right]^{2}
$$

pour $n=n^{\prime}$. Il vient donc

$$
\mathrm{I}=\frac{\mathrm{I}}{2 \mathbf{T}^{2}} \sum_{1}^{\infty}\left\{\left[\varphi_{(}\left(\theta_{n}\right)\right]^{2}\left(\mathbf{A}_{n}^{2}+\mathrm{B}_{n}^{2}\right)\right\}=\sum_{1}^{\infty} \mathrm{J}_{n},
$$

en désignant par $I_{n}$ l'intensité lumineuse que produirait au point $\mathbf{P}$ le mouvement simple (6), s'il existait seul ( $\left.{ }^{1}\right)$, d'après la formule ( 7 ).

Ainsi l'intensité lumineuse en un point quelconque, dans le cas d'une lumière constante, est la somme des intensités que donnerait, pris séparément, chacun des mouvements simples dont l'expression est donnée par les formules (4) et (6), dans lesquelles on doit supposer $\mathrm{T}$ très grand.

Ainsi se trouve justifié le principe physique de l'indépendance des rayons d'espèce différente, qui, au premier abord, paraissait en contradiction avec ce fait que tous les mouvements vibratoires se superposent dans le même milieu élastique.

4. Analyse spectrale. - Supposons que le système optique considéré soit un appareil pouvant produire un spectre. Soit le point $P$ pris sur ce spectre, et soit $\theta_{0}$ la période vibratoire du mouvement lumineux simple qui viendrait former son image au point $P$. La théorie ondulatoire nous apprend que la fonction $\varphi_{(}\left(\theta_{n}\right)$ n'aura de valcur sensible que pour les valeurs de $\theta_{n}$ comprises entre $\theta_{0}-\varepsilon$ et $\theta_{0}+\varepsilon, \varepsilon$ étant très petit vis-à-vis de $\theta_{0}$, et d'autant plus petit que le spectre sera plus pur, et le pouvoir optique de l'ap-

( ${ }^{1}$ Le terme intensite est pris comme synonyme de force vive moyenne. 
pareil plus grand. Ainsi le mouvement vibratoire au poinı $\mathrm{P}$ résultera de la superposition de mouvements simples ayant des périodes très voisines de $\theta_{0}$; ce mouvement sera donc un mouvement sensiblement régulier dont la période sera très voisine de $\theta_{0}$; et la couleur de la lumière au point $\mathrm{P}$ sera celle du rayon lumineux simple qui viendrait former son image en ce point.

Le nombre des mouvements simples pour lesquels $\theta_{n}$ est compris entre $\theta_{0}-\varepsilon$ et $\theta_{0}+\varepsilon$ est d'ailleurs proportionnel à $T$, d'après $(\tilde{)})$. Comme les raisonnements précédents ne sont rigoureux qu'à la limite, pour de très grandes valeurs de $\mathbf{T}\left({ }^{1}\right)$, on doit toujours regarder ce nombre comme très grand. L'intensité aux différents points du spectre dépendra de la loi suivant laquelle l'intensité de ces mouvements simples varie avec leur période, c'est-à-dire des quantités $\mathrm{A}_{n}$ et $\mathrm{B}_{n}$.

Pour que la lumière incidente parût homogène à l'analyse spectrale, il faudrait que ces mouvements simples se réduisissent à un seul, ou du moins à un groupe resserré de périodes extrêmement voisines; si cette condition n'est pas remplie, il y aura élargissement de la raie ou formation d'un spectre continu, chaque mouvement simple venant former son image en un point différent du spectre. L'examen des formules (4) montre que cette condition n'est réalisée que si le mouvement incident constitue lui-même un mouvement simple, d'une régularité parfaite. Ainsi les irrégularités et les perturbations quelconques du mouvement incident produisent l'élargissement des raies spectrales et les spectres continus qui les accompagnent d'ordinaire; ce point de vue sera développé dans un travail ultérieur.

\section{Interférences et régularité du mouvement lumineux. -} Supposons maintenant que le système optique considéré soit un appareil interférentiel.

Les mouvements simples donnés par la formule de Fourier étant d'une régularité absolue et d'une durée indéfinie, les interférences se produiraient pour chacun d'eux, sans aucune perturbation pos-

( ${ }^{1}$ ) A cause de l'erreur qui peut exister au commencement et à la fin de l'intervalle $2 T$ ( $n^{\circ} 2$ ), dont nous n'avons pas tenu compte dans le calcul de I. 
sible. Nous avons vu d'ailleurs que l'intensité lumineuse réelle est la somme des intensités que donnerait chaque mouvement simple. Ainsi le phénomène réel sera formé par la superposition des systèmes de franges que donnerait chaque mouvement simple pris séparément. Suivant que ces systèmes seront concordants ou discordants, il y aura ou non des franges visibles. Il y a deux cas principaux à examiner.

Premier cas. - La lumière incidente est plus ou moins homogène, et l'on produit les franges sans le secours d'un appareil spectral. Soient $\theta_{1}$ et $\theta_{2}$ deux limiles qui comprennent les périodes des mouvements simples qui constituent le mouvement incident. Si les systèmes de franges correspondant aux périodes $\theta_{1}$ et $\theta_{2}$ ne sont pas discordants, c'est-à-dire s'ils diffèrent de moins d'une demi-frange, il en sera de même a fortiori pour les périodes intermédiaires, et l'on aura des franges visibles. Mais l'examen spectroscopique de la lumière incidente permet de déterminer les limites $\theta_{1}$ et $\theta_{2}$; ainsi il existe une relation nécessaire entre le degré d'homogénéité de la lumière, tel qu'on le détermine au spectroscope, et la visibilité des franges d'interférences dans telle condition donnée, sans qu'il soit nécessaire de faire d'hypothèse sur la régularité du mouvement. Cette relation est due à ce que la régularité plus ou moins grande du mouvement détermine l'écart des limites $\theta_{1}$ et $\theta_{2}$, comme nous l'avons vu au $\mathrm{n}^{\circ} 4$.

Second cas. - La lumière incidente est blanche, ou susceptible de donner un spectre continu, et l'on fait usage d'un appareil spectral pour observer les franges, soit par la méthode de MM. Fizeau et Foucault, soit en isolant une portion du spectre, qui sert de source de lumière. Dans tous les cas, on obtient la superposition des franges produites par les mouvements simples dont les périodes sont comprises entre deux limites $\theta_{1}$ et $\theta_{2}$, limites qui dépendent, non plus de la lumière incidente comme dans le cas précédent, mais de l'appareil lui-même, et du point considéré, la fonction $\varphi\left(\theta_{n}\right)$ n'étant différente de zéro qu'entre ces limites. Si ces systèmes de franges sont concordants, on aura des franges visibles. En particulier, dans l'expérience de MM. Fizeau et Foucault, les franges seront visibles toutes les fois que lc 
spectre sera assez pur et la lunette assez puissante pour qu'on puisse séparer deux franges voisines.

Ainsi l'existence de franges d'interférences à grande différence de marche, dans le cas des sources à spectres continus et de la lumière blanche, n'implique nullement la régularité du mouvement lumineux incident. Cette régularité existe dans le spectre, mais c'est l'appareil spectral qui la produit, en séparant plus ou moins complètement les divers mouvements simples qui, jusque-là, n'avaient qu'une existence purement analytique.

Pour considérer un cas extrême, à titre d'exemple, on peut regarder la lumière blanche comme formée par une suite d'impulsions tout à fait irrégulières, ou de vibrations sans cesse troublées, analogues au mouvement de trépidation qui, pour quelques physiciens, constitue le mouvement calorifique. Cette hypothèse assez vraisemblable permet d'expliquer simplement la continuité parfaite du spectre, assez difficile à comprendre si l'on considère la source lumineuse comme produisant des séries distinctes de vibrations régulières. 